\title{
Correction of Galileo Energetic Particle Detector, Composition Measurement System High Rate Data: Semiconductor Dead Layer Correction
}

\author{
Z. Lee-Payne ${ }^{1}$ P. Kollmann ${ }^{2}$ M. Grande ${ }^{1} \cdot$ T. Knight ${ }^{1}$
}

Received: 2 November 2018 / Accepted: 23 November 2019 / Published online: 8 January 2020

(C) The Author(s) 2020

\begin{abstract}
The Energetic particle detector (EPD) on-board the Galileo orbiter measured ions in the $\mathrm{keV}$ to $\mathrm{MeV}$ energy range in Jupiter's equatorial plane, generating a unique data set. However, as a result of its time in the highly hostile Jovian radiation environment the EPD and its composition measurement system (CMS), suffered damage to its silicon semiconductor detectors. This resulted in misidentification of ion species and an underestimation of fluxes. We use in-flight measurements to understand the nature of the damage and how much it has affected the data. A method of mass and energy identification and reallocation was developed to identify and correct affected data thus improving the usability and accuracy of the Jovian data sets. This study aims to calculate the extent of the damage to the main CMS detector, $K_{T}$; estimating the build-up of a non-sensitive dead layer on its surface. By the end of mission this dead layer has increased and is estimated to top out at $0.35 \pm 0.02 \mu \mathrm{m}$. A corrected set of data is produced, which fits better within expected boundaries, and will be made available for future use.
\end{abstract}

\section{Introduction}

Jupiter's magnetosphere, carves out a huge magnetic cavity for itself and its satellites (Bagenal 2007; Bagenal et al. 2004). This cavity contains the hot low-density plasma from Jupiter and its four largest moons, whilst deflecting the solar wind. Unlike the Earth's magnetosphere, which is solar wind driven, the magnetosphere of Jupiter is rotationally dominated with internal plasma sources, the main heavy ion being sulphur from Io (Bagenal and Delamere 2011). However, a small amount of the solar wind seeps in through reconnection making ion composition richer.

Note by the Editor: This is a Special Communication, linked to the Topical Collection on the Galileo mission published in Space Science Reviews (https://link.springer.com/journal/11214/60/1).

Z. Lee-Payne

zleepayne@gmail.com

1 Aberytwyth University, Aberystwyth, UK

2 Johns Hopkins University Applied Physics Laboratory, Laurel, MD, USA 
The frozen-in plasma is pulled along with the fast-rotating magnetic field, and is confined by centrifugal forces to the magnetic equator (Chané et al. 2013; Huddleston et al. 2000). The plasma disk or magnetodisc is the name given to these equatorially confined particles, a key component of Jupiter's magnetosphere; however, it coexists with powerful electron and ion radiation belts which make its measurement difficult. The following research focuses on the effect of radiation damage to the detector. Radiation damage on the detectors accumulates over time, resulting in their degradation, which then affects measurements everywhere in the Jovian system. This radiation induced degradation on energetic ion composition measurements, and a correction to them, is the subject of this work.

The majority of the material that fills the plasma disk comes from the moon Io; this is the primary source of Sulphur ions in the system. Helium ions are sourced from Jupiter's ionosphere, with the other large icy moons being a source of oxygen ions. The last major species, hydrogen ions, are sourced from the solar wind as described previously. Each source has a relatively distinct signature in EPD data (Bagenal 2007; Jun et al. 2005), with the most complex compositional signatures coming from the Galilean moons. The surfaces of Io and Europa have very little protection from the plasma disk that they are moving through and this leads to material being lifted, or sputtered, from the surface. These particles are then picked up by the magnetic field and combine into the existing plasma of the magnetodisc.

The energy of the ions measured in this detector is expected in the range of $100 \mathrm{keV}$ up to $5 \mathrm{MeV}$. Ion data has been measured by all nine spacecraft that have visited the Jovian system, including the Galileo Orbiter which took measurements from 1995 until 2003 (Jun et al. 2005). There is a higher density of particles near to the center of the disk due to centrifugal confinement. This contributed greatly to the data degradation during the later stages of the mission.

\subsection{The Energetic Particle Detector (EPD) Composition Measurement System (CMS)}

The EPD (Paranicas et al. 2007; Williams et al. 1992) flown on the Galileo orbiter consists of two main instruments: The CMS and the Low-Energy Magnetospheric Measurements System (LEMMS).

The EPD aimed to provide major extensions to the Jovian energetic particle data base, expanding on the data taken by the Pioneer and Voyager missions. Its initial two-year mission lifetime allowed both a direct measure of time variations of plasma content and behavior in the Jovian magnetosphere and a significantly larger spatial sample of the system than before. The low-energy thresholds of the EPD effectively closed the energy gap between plasma and energetic particle measurements (Williams 1996, 1997; Williams et al. 1992).

The CMS is composed of multiple detectors and a time of flight (TOF) measurement system. The ion composition of a plasma is measured by recording both the energy and the time of flight of the particles allowing the mass of the particle to be determined. CMS consisted of three telescopes. We consider only the $\mathrm{TOF} \times \mathrm{E}$ telescope in this study; the two $\triangle \mathrm{E} \times \mathrm{E}$ telescopes are beyond the scope of this paper. Figure 9 of Williams et al. (1992), illustrates a schematic of the full CMS detector. Mounted toward the main viewing direction of the EPD, the TOF detector consists of a parylene foil, followed by a main silicon semiconductor detector (SSD). The start/stop signals (and time of flight between them) are produced by secondary electrons emitted from the parylene start foil and electrons emitted from the SSD surface. The TOF combined with the distance between the foils gives the velocity $v$ of the moving particles. Once the particles have passed through the foils they impact into a 
Fig. 1 TOF vs. Energy observed in detector KT. Discriminator levels defining the indicated TOF rate channels (solid lines), predicted TOF vs. $E$ track locations (Grey dashed lines); blue section of these loci highlight where the tracks turn. Data from accelerator calibrations can be found for the same diagram in Williams et al. (1992). The channel names are indicated within each channels boundaries in relation to element expected: Iron: TH1, Sulphur: TS1, TS2, TS3, Oxygen: TO1, TO2, TO3, TO4, Helium: TA1, TA2, and Hydrogen: TP1, TP2, TP3

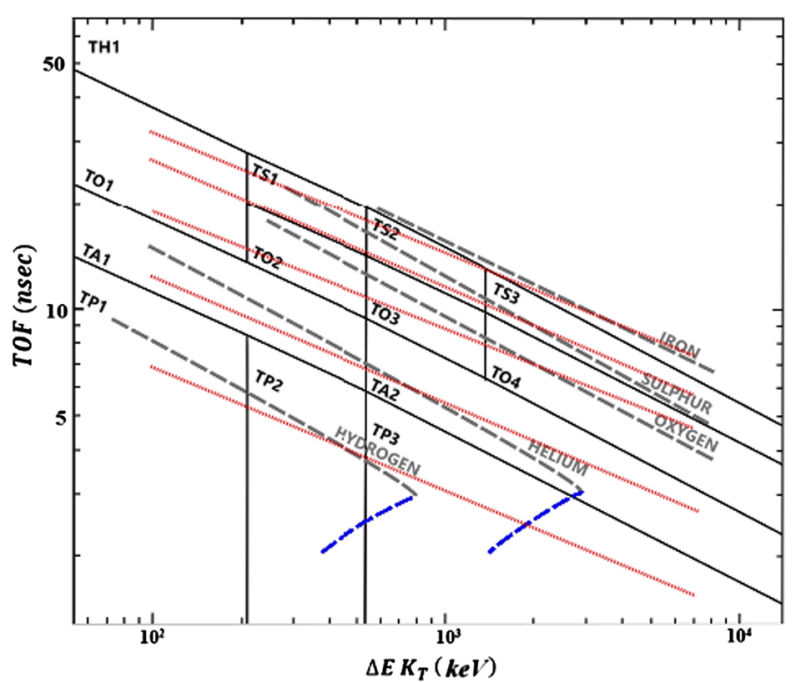

silicon semiconductor detector, $K_{T}$. The energy, $E$, that the ion deposits in $K_{T}$, combined with the velocity $v$ of the particle allows the calculation of the mass $\mathrm{m}$. To first order, when the particles do not lose significant energy in the foils and dead layer, and the particle is not relativistic, the relation is $E=\left(m v^{2}\right) / 2$. Figure 1 shows expected data, taken during ground readings.

The $K_{T}$ detector is a $12.6 \mu \mathrm{m}$ thick silicon semi-conductor detector. These are commonly used for radiation and particle detection, the close band gap properties attributed with semiconductors makes it ideal for detecting ionizing radiation. The silicon used in detectors is not pure; most often it is doped into $\mathrm{N}$ and $\mathrm{P}$ sections. $\mathrm{N}$ doping adds a loose electron in the valance band. $\mathrm{P}$ doping adds an almost full shell of electrons to the conduction band, this in effect adding a 'hole' for the loose electrons in the $\mathrm{N}$ doped band to be excited into. When a $\mathrm{P}$ and $\mathrm{N}$ doped semiconductor are next to each other, the free electrons and open electron holes intermingle and a conductor-like interaction happens. That is until a bias is applied i.e. a 'reverse' voltage applied to the $\mathrm{N}$ doped side of the semiconductor. This bias reduces the size of the PN junction holding the electrons in the P doped material and the keeping the holes to the $\mathrm{N}$ doped material. This forms a energy-sensitive volume in between (Grupen and Shwartz 2011).

When an incoming particle hits the detector, the particle transfers its energy into excitation of electrons from the valence into the conduction band. The energy transferred allows a proportional number of electrons to excite and such causes a current to temporarily flow, counteracting the negative bias. This counteraction is measurable as a drop in the bias voltage with an electronic amplifier. The voltage drop is to first order proportional to the kinetic energy of the impacting particle as it hits the detector.

However, as each particle enters the sensitive volume of the detector the energy imparted denatures the silicon doping a small amount, usually in a way that effects the excitation and the separation of electrons and holes, in the outermost surface of the detector. This increases the thickness of the non-sensitive dead layer on the front of the detector, to above its initial manufactured value. The deeper the dead layer grows into the silicon, the lesser the damage by the particles is, as particles will have already lost energy in passing through the dead layer. This implies that the growth of the dead layer will slow down and its thickness will 
saturate eventually, a behavior that we also observe in the CMS data discussed below. While the dead layer does not affect the operation principle of the sensitive volume in its ability to measure energy, the dead layer does decrease the size of the sensitive volume and the energy measured. As the dead layer thickens, the energy lost by the particle before reaching the sensitive volume increases.

In the case of helium and hydrogen the most energetic particles can pass through the sensitive layer, which causes a reduction in the amount of energy they deposit and thus the measured energy. This effect can be seen in the PHA plot (Fig. 1), where the hydrogen and helium loci turn back on themselves at the higher energy end as indicated by the blue section.

Many studies (such as Mauk et al. 2004 and Clark et al. 2016) that have used the EPD data, noted the inbuilt precautions and safeguards to deal with a build-up of a dead layer which were implemented in the instrument. However, the extent of the decay exceeded the expected amount, and many using the data have elected to use only the first two years out of eight for data analysis, as it is easily correctable. Studies on precise intensities or species abundance ratios, relying on data late in the mission may be in need of updating.

\subsection{PHA Plots}

Each detected ion can be represented as a point in a plot of deposited energy vs. TOF, (Fig. 2). The relation between these quantities is determined by the ion mass, which means that points in such a plot are not randomly distributed but follow tracks/loci. The number of tracks is determined by the number of particle species in the Jovian system. These measured loci should match the predicted positions shown in Fig. 1.

A valid TOF event requires a triple coincidence: the start MCP event, followed within $60 \mathrm{~ns}$ by a stop MCP event, and finally a coincident $K_{T}$ energy pulse. Each TOF event is also filtered through a hardwired priority system on board the instrument. This is designed to stop the far higher flux of protons from overwhelming the rarer oxygen and sulphur particles. There are 4 main priorities; the top TH1 box has its own priority to highlight the rare species expected here. The next two cover priorities cover the oxygen and sulphur channels, splitting into priority 2 (high energy oxygen and sulphur in channels TO3-4, TS2-3) and 3 (lower energies in channels TO1-2, TS1). The final priority fills the remaining channels covering Helium and Protons.

The priority system orders each event transmitted based on the last event sent. As each particle is measured in the system its priority is recorded. Then approximately every $0.2 \mathrm{sec}-$ ond window one particle's data is transmitted, specifically the particle with the next highest priority to the one previously. Whilst this means that there should equal numbers of all of the priorities, in a given window a particle of the required priority may not have been recorded in the time gap, in which case next highest priority particle is sent.

The PHA plots are an accumulation of data points over time and over the priorities, plotted on dual log scales, with each channel normalized to keep the boundaries between the priorities smooth.

Figure 2 shows a comparison between measurements taken at different times. Figure 2 a is from the first year of the mission from 29th Sep 1996 until 9th October 1996. This data is as expected; the main element mass loci fall within the channel boundaries in the mass discriminator lines. This is not the case in the data as the mission progresses, as can be seen in Fig. 2b. The locations of the loci and the count rates are the most visible changes. 


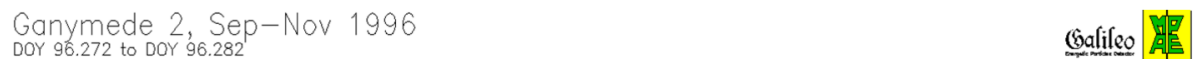
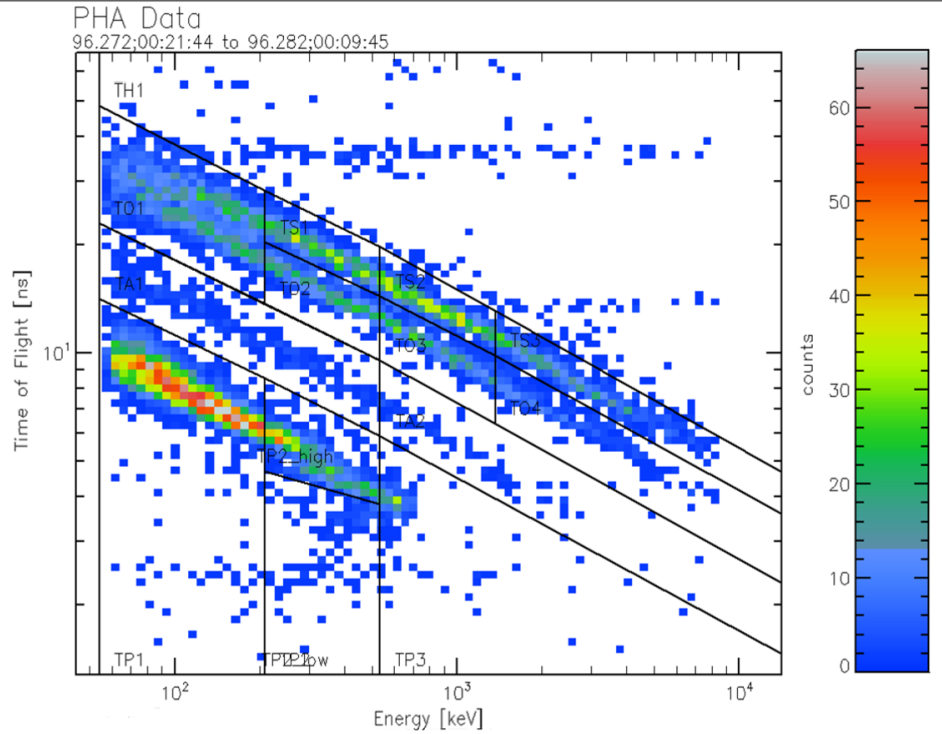

Amalthea 34, Oct-Nov 2002 DOY 02.295 to DOY 22.305

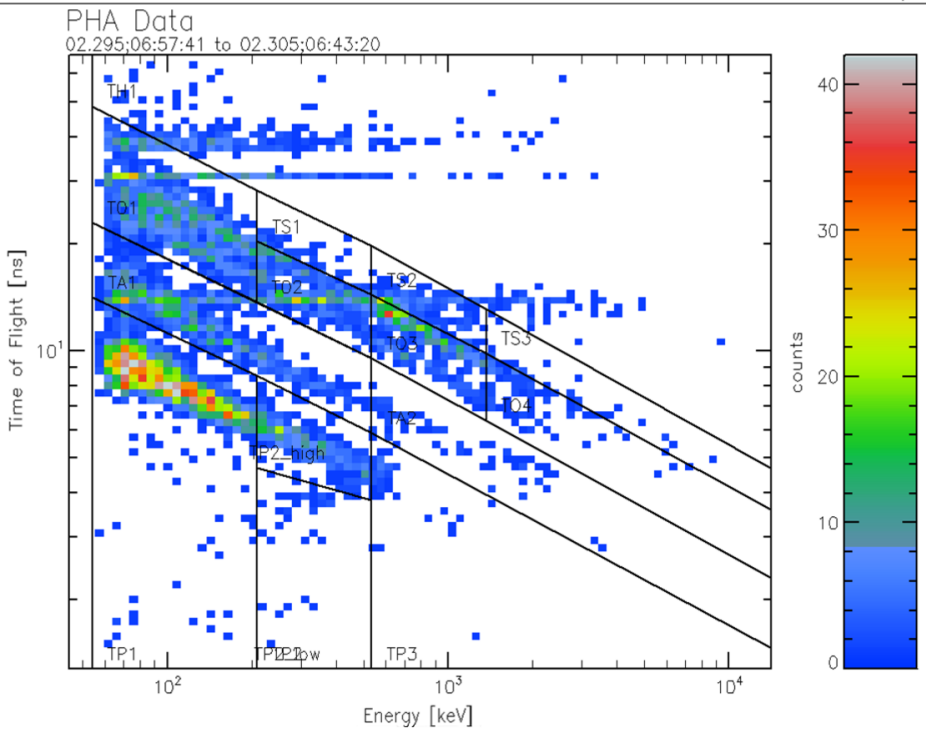

Fig. 2 PHA event data as a function of TOF and energy. (a) data taken in 1996 from day 272 to 282, (b) data taken in 2002 from day 295 to 305

Notable differences include:

1. The location of the mass loci; The Sulphur line in particular has dropped completely out of the TS channels and is being recorded in the TO channels. The same is also happening with the oxygen lines and to a lesser extent the hydrogen and the helium. The heavier mass element lines are being affected more. 
Table 1 Energy loss of different ion species (lines) in silicon dead layers of different thickness (columns). The energy loss is based on Ziegler et al. (2010) and provided in $\mathrm{keV}$

\begin{tabular}{llrrrrrrrrr}
\hline Element & $0.0 \mu \mathrm{m}$ & $0.05 \mu \mathrm{m}$ & $0.1 \mu \mathrm{m}$ & $0.15 \mu \mathrm{m}$ & $0.2 \mu \mathrm{m}$ & $0.25 \mu \mathrm{m}$ & $0.3 \mu \mathrm{m}$ & $0.35 \mu \mathrm{m}$ & $0.4 \mu \mathrm{m}$ & $0.5 \mu \mathrm{m}$ \\
\hline Iron & 0 & 64 & 123 & 182 & 241 & 300 & 359 & 418 & 477 & 595 \\
Sulphur & 0 & 38 & 80 & 122 & 164 & 205 & 247 & 289 & 331 & 414 \\
Oxygen & 0 & 17 & 40 & 63 & 86 & 109 & 132 & 155 & 178 & 224 \\
Helium & 0 & 4 & 9 & 15 & 21 & 27 & 33 & 39 & 45 & 57 \\
Hydrogen & 0 & 2 & 7 & 12 & 17 & 21 & 26 & 31 & 36 & 46 \\
\hline
\end{tabular}

2. Increase in anomalous data; at approximately 15 and $30 \mathrm{~ns}$ TOF across the whole of the energies there is a solid line of data that is different from the others surrounding. This is most likely a fault in the TOF detector.

3. Difference in the turning point of the helium and hydrogen loci (blue section of the element tracks in Fig. 1): The energy at which these lines turn is another measure of the dead layer. This could be used to calculate the dead layer thickness; however, this method is less accurate and we have not used it in this study.

When using the earlier data, which is less effected by sensor degradation, simple methods are applicable for making appropriate adjustments for the data as described in the appendix of Mauk et al. (2004). However, as the mission progresses, the ratio of data against data gap from the EPD widened. These gaps add to the uncertainty in adjusting the data correctly as the flux responsible for the dead layer growth in these intervals is not measured.

\section{Thickness of Semiconductor Dead Layer}

\subsection{Method of Estimation}

To re-analyze the data, the first step is to assess the thickness of the dead layer as the mission progresses. This is done using a direct comparison of the measured loci (lines over-plotted in Fig. 3a-e), against expected loci based on a TOF-energy relation for each element applicable; we consider this our base calibration tracks (shown in Fig. $3 \mathrm{f}$ as the red loci). Figure $3 \mathrm{f}$ shows the mismatch between our base calibration and observations during the ground calibration (Williams et al. 1992, and Fig. 1). The typical process for instrument ground calibration is to take measurements with an identical detector, analyze the results and then adjust the limits of the instrument to account for the performance of the real detector. Our base calibration lines show how much the boundaries were adjusted when compared to the measured ground calibration lines.

When a particle travels through a given thickness of material it deposits the energy shown in Table 1. The values are calculated using the Stopping Ranges in Matter (SRIM) (Ziegler et al. 2010) collection of software packages; specifically, TRIM stopping range tables. This is the maximum energy that a particle can lose to the dead layer. A particle which has sufficient energy to pass through the dead layer, will lose less energy than this, maximum energy, which is also the minimum energy required to penetrate the layer.

For simplicity only this maximum energy loss is used as a variable. The use of this means there will be some discrepancy in the final correction, in that the higher energy particle correction will be less accurate as the particles lose a greater amount less energy than expected. 


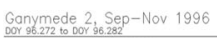

a)

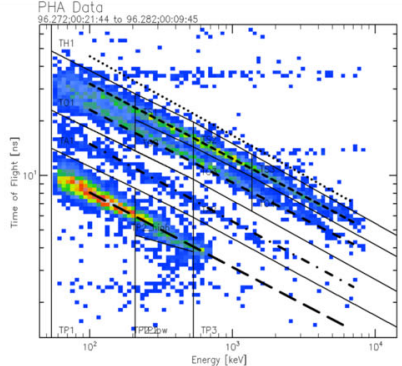

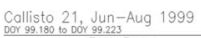

c)

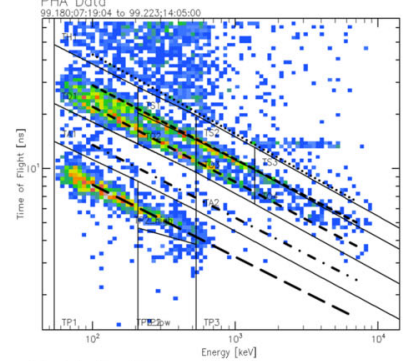

Ammatheo 34. Oot-Nov 2002

e)
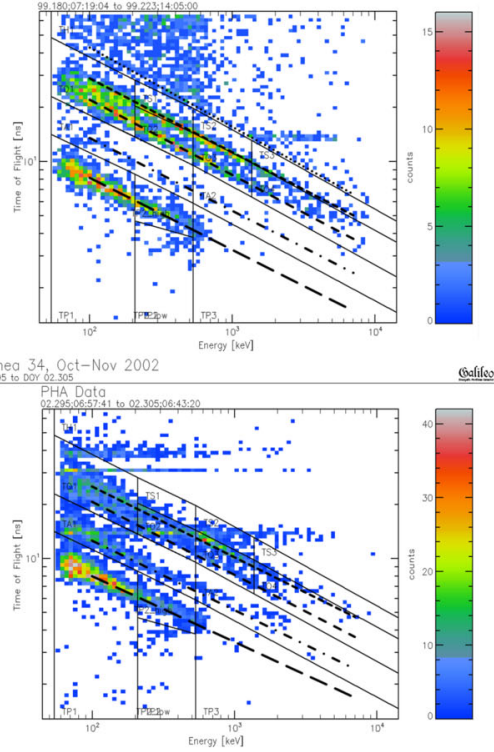

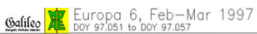
Gosflec)

b)

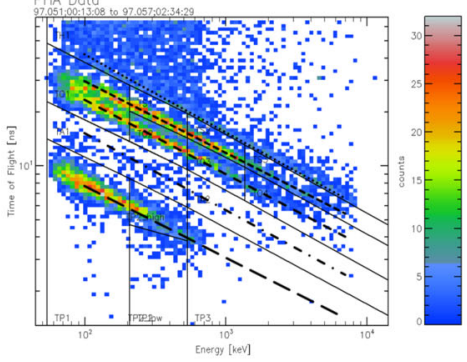

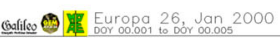

d)

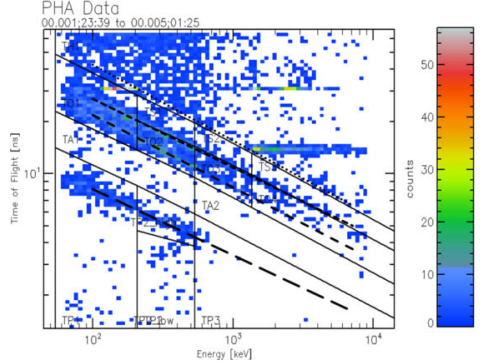

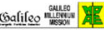

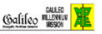

f)

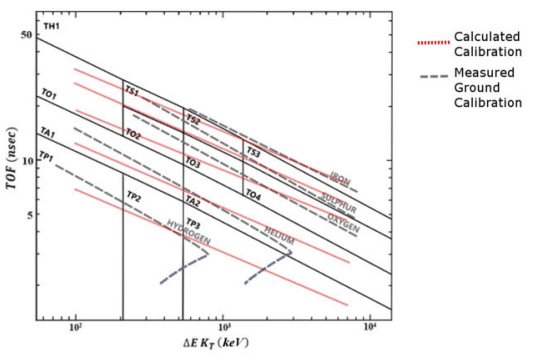

Fig. 3 PHA plots of original data overlaid with loci marking the currently observed energy track. Data sets from: (a) 1996 day 272 to 282, (b) 1997 day 51 to 57, (c) 1999 day 180 to 223, (d) 2000 day 1 to 5 and (e) 2002 day 295 to 305 . Panel (f) is the same as Fig. 1 but overlaid with the base calibration tracks (red dashes)

However, the higher energy particles are less effected by the dead layer, thus the effect is less obvious. This also allows for keeping consistency in variables over the correction is preferable and will be discussed in detail later.

Using the values in Table 1 we shift the tracks of the base calibration in energy based on a thickness of dead layer. We keep the TOF constant. This generates a set of expected track locations for a set of dead layer thicknesses; these are compared with observed data.

The observed data comes from 5 time periods across the mission, we will determine the dead layer thickness at each period. These periods are selected as they provide sufficient event rates to build up a clear PHA plot in a short time interval. All 5 PHA plots are shown in Fig. 3a to e. The observed tracks (dashed lines) for each plot are best drawn by eye.

Comparison of the observed data with the sets of expected track locations gives the dead layer buildup. To achieve this, we apply a Chi Squared test. The chi squared compares a set of points along each loci with the results shown in the following section. 
Fig. 4 Increase in dead layer thickness over time, determined by best fit data. The smaller error shading (dark blue) shows the percentage difference in the next closest fit of dead layer. The larger error shading (light blue) shows the percentage difference in the second next closest fit of dead layer

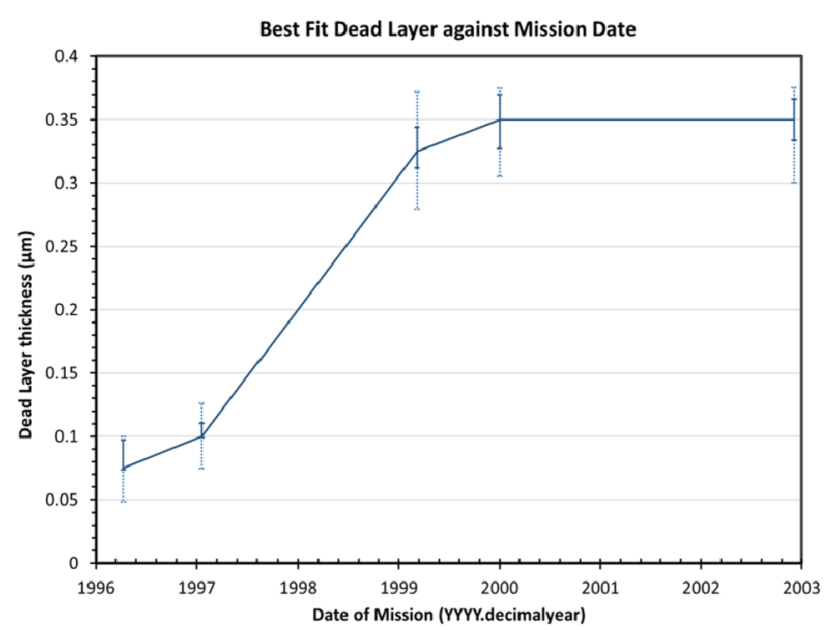

\subsection{Estimation Results}

The first chi squared results are shown in Fig. 4 as the estimated growth of the dead layer thickness. It identifies a value of the dead layer around the $0.35 \pm 0.2 \mu \mathrm{m}$ thickness at the end the mission in late 2003. The error shading indicates how much the fit would need to change before a neighboring thickness value would be considered as providing the best fit.

The values of the 1996 (early Jupiter phase) dataset match best with the dead layer thickness of $0.0750 \mu \mathrm{m}$. This is consistent with the initial dead layer of the un-degraded instrument as described in Williams et al. (1992) and any dose that be have been incurred during cruise. The ground calibration needs to be taken into account when estimating the actual dead layer. This is done by using the $0.075 \mu \mathrm{m}$ dead layer set as the new base calibration line. Using the values in Table 1 we shift the new base calibration in the same manner for set thicknesses of dead layer, as this will affect the rate at which the dead layer is estimated to be increasing during the first years of the mission. Without this adjustment the data would be over-corrected in these first years. The chi squared values are then re-tested, and the new estimated dead layer thickness are thus as seen in Fig. 5 with the values given in the Appendix (Table 2).

The build-up of the thickness matches well with the phases of the mission and the relative intensities and densities of the particle environment the spacecraft is traveling through. In the initial phase of the mission, insertion in 1995 to orbit E11 in 1997, the spacecraft only ventured towards the inner moons 3 times, remaining in the less energetic areas around Callisto and Ganymede. The build-up of the dead layer is gradual. After this phase the extended mission focused on Europa where, until 2000, it completed 10 flybys of Europa and Io; this section of the mission can be clearly seen in the rapid increase in the dead layer build up between the 1997 and 1999 data sets (Fig. 5 shows the dates of the flybys as vertical overlays). Being closer to Io and Europa the particles are at far higher energy flux and more likely to be of a higher mass species, all of which will contribute to a faster build of the dead layer.

The final phase of the mission, beginning in 2000 until impact in 2003, includes flybys of all 4 moons and the smaller moon Amalthea. At this point of the mission the dead layer is sufficiently thick to absorb a significant fraction of the incoming particle energy and hence 
Fig. 5 Updated increase in dead layer over mission years determined by best fit data, accounting for instrument calibration. Additional vertical lines indicate Europa flybys. The smaller error bars show the percentage difference in the next closest fit of dead layer. The larger error shows the percentage difference in the second next closest fit of dead layer

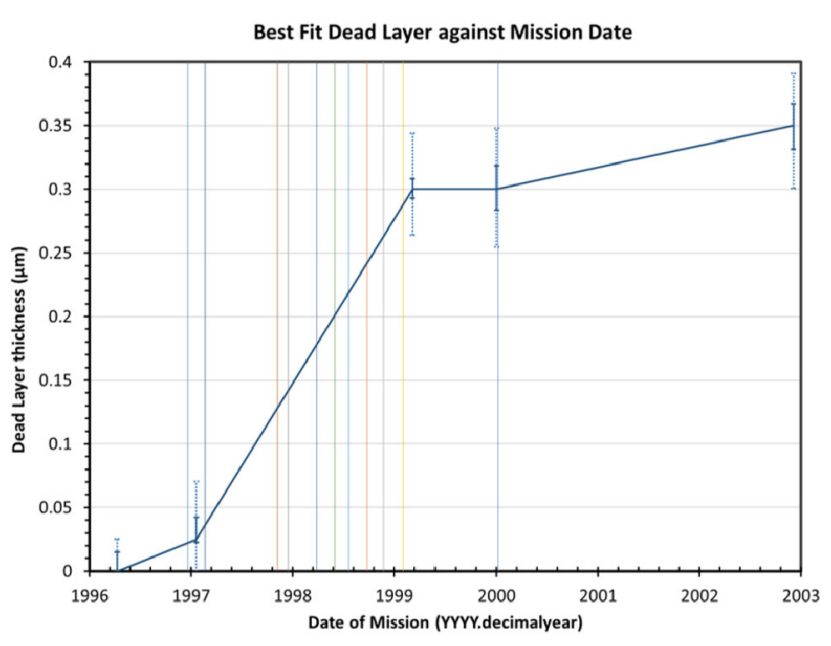

the dead layer thickness begins to saturate. The upward trend in this phase most likely comes from the three orbits of Io at the very end of the mission. Here the higher concentration of sulphur due to the close proximity to Io, combined with higher radiation fluxes, would have produced a significant detector decay, counteracting the saturation effects.

Between the 1997 and 1999 data sets the increase of the dead layer is considerable. Data from the mid-point in this time period would allow further insight into how the growth progressed. Unfortunately, the PHA plots at this time are sparsely populated and loci are difficult to identify. This can be seen for example in the helium channels of Fig. 3d.

\section{Correction of the Data Set}

For each measured PHA event, we assume that the TOF and time stamp are correct. From the timestamp we can assign a dead layer thickness using the results of the previous section. We then test all the possible masses, in nucleon increments, greater or equal to the original assigned mass and calculate the initial energy for each test mass from the TOF. These energy-mass combinations are propagated through the dead layer and detector to produce a set of detected energy measurements using values as in Table 1. This set of possible mass energy combinations is compared to the actual measurement. The mass which gives the closest agreement is selected.

In this way we produce the mapping shown in Fig. 6, 7, and 8, where the corrected points on the right hand panel correspond to the best fit for the raw measured points on the left hand panel.

Our procedure accounts for species that are not expected at Jupiter. This is required because the measured counts do not line up exactly on a sharp track but are instead spread out due to scattering and straggling in the instrument, as well as due to measurement uncertainties. This consideration of test masses not physically abundant at Jupiter, keeps the correction consistent between each channel and predicts the errors in the species assigned to each channel. The correction of the count rate data collected for each individual channel, will form the content of a follow on publication. 

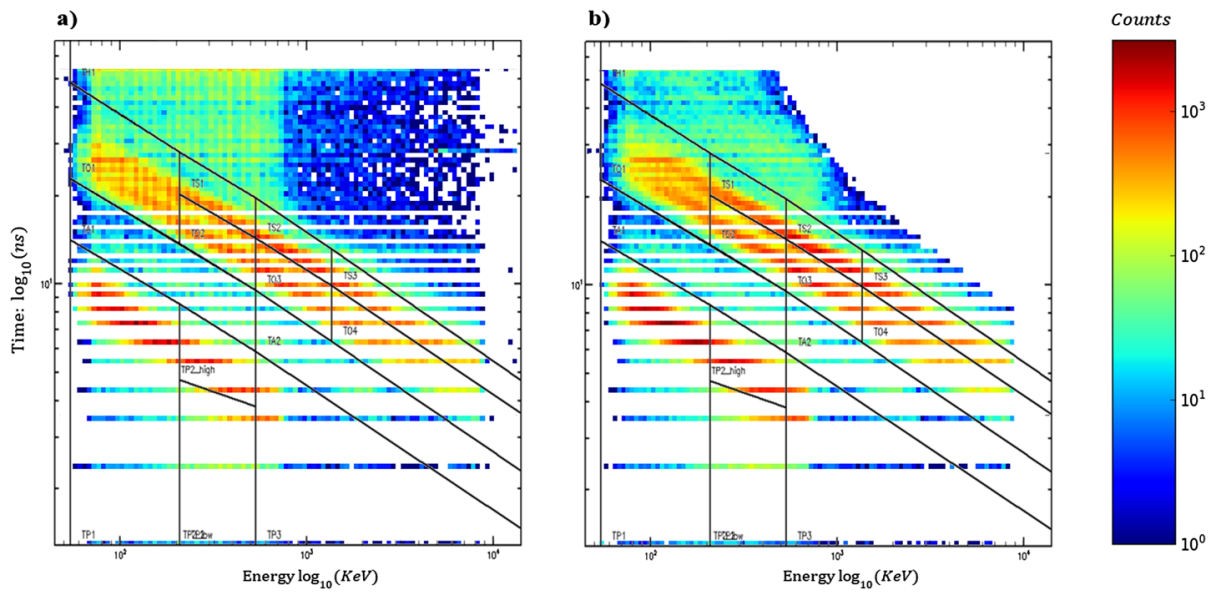

Fig. 6 Data from pre 1999 mission with the original channel boundaries overlaid. (a) Original data. (b) Corrected data
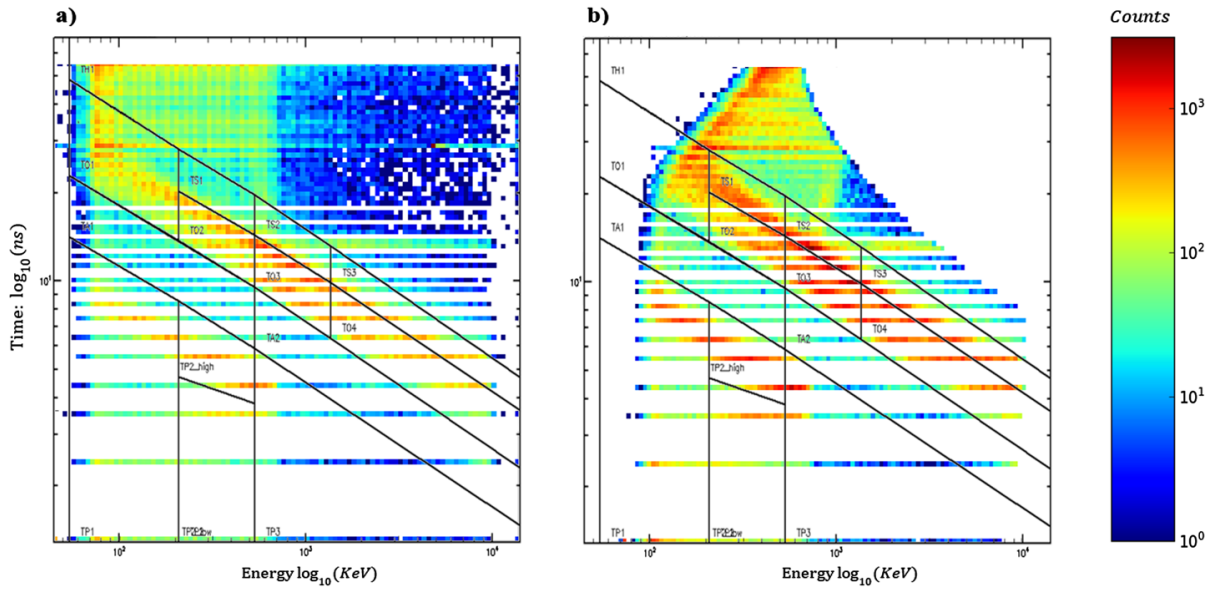

Fig. 7 Data from post 1999 mission with the original channel boundaries overlaid. (a) Original data. (b) Corrected data

\section{Corrected Data Set}

\subsection{Results}

The main discrepancies in the data are seen in the later years of the mission; this is easiest to see in the data after 1999 (see Fig. 2). The pre-1999 data is mostly still within the original bounding boxes for each of the elements. After 1999 the Sulphur ion signal is no longer present in the TS channels has moved and begun contaminating the TO channels.

Figure 6 shows data all the data prior to 1999, the format of this figure and the following Figs. 7 and 8 shows the original untouched data (a) along with the correction of the same data (b). The data has been re-plotted in both cases for ease of comparison. The original 

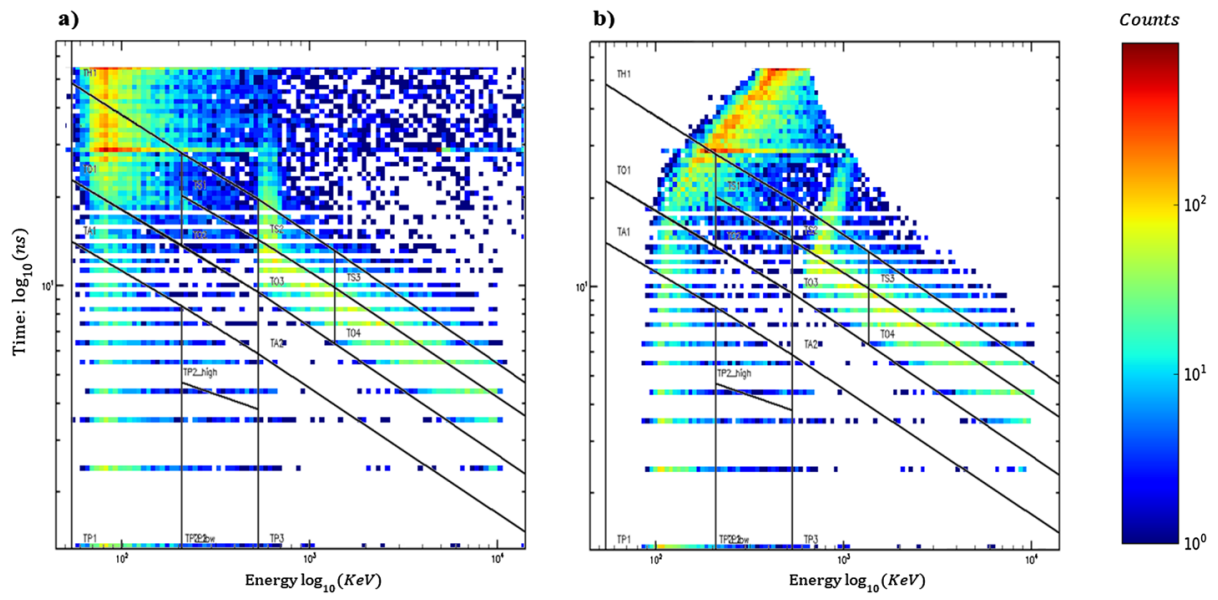

Fig. 8 Data from 2002 through to 2003 with the original channel boundaries overlaid. (a) Original data. (b) Corrected data

boundaries of the channels have been plotted overtop to show the movement between the boundaries.

In the corrected 1999 data (Fig. 6b) the loci for oxygen ions and sulphur ions are more distinct. The position of the corrected loci is now more consistent with the original TS channel boundaries, and its overall grouping is narrower, though there is not much overall change. This reexplains why the data from the first few years of the mission was more commonly used than later data.

Figure 7 shows the original and corrected data from 1999 to the end of the mission. This is the data that was most effected by the increase in the dead layer, where the correct location of data, relative to the channel boundaries, is considerably improved. There is some spread in the data presented due to gaps in the TOF caused by the log scaling. This limits how much the correction appears to have improved that data.

Figure 8 isolates the worst period of data from the mission and the point in which the correction is affecting the data the most. The effect this short time-span of data has on the overall mission is quite considerable and illustrates how, despite the improvement achieved, the final year of data should still be treated with considerable care.

\subsection{Discussion}

Estimating the thickness of the dead layer is complicated and some assumptions must be made. The first is in accounting for the ground calibration of the instrument. We use a value of $0.075 \mu \mathrm{m}$ to give a best fit to the ground calibration. Using the chi-squared method of fitting data offers a good approximation. A better way to do this might be to use the data generated during the ground calibration tests (Fig. 2), however more information would be needed such as the energy and fluxes of the ions tested.

Whilst this does not affect the validity of the thickness estimation, the value produced should be considered a lower bound of dead layer thickness, and in particular the relative of journey phase and ground degradation. This consideration will also account for any discrepancies in the angle of impact of the incoming particles, and the small change in energy measured that would come from the $18^{\circ}$ collimator angle. 
The lack of ions in the top left-hand corner of Figs. $7 \mathrm{~b}$ and $8 \mathrm{~b}$ implies that the modeled late mission dead layers does not allow these low energy ions to penetrate to the sensitive region of the detector. The lack of ions in the upper right reflects the fact that counts with high deposited energy but low velocity observed in Fig. 8a cannot be modeled as realistic particles in a realistic detector geometry. These counts are thus clearly nonphysical (they would have extremely high masses) and are thus likely the result of spurious coincidences. Particle misidentification can occur with errors in the triple coincidence; for example, if START and SSD signal are valid but the STOP pulse is not recoded and instead a particle that comes a later provides the STOP. Similarly, high count rates in the top left of Figs. 7 and 8 below $100 \mathrm{keV}$ before correction can be attributed to this noise.

There is also some uncertainty in the correction itself, this primarily comes from the values used in Table 1. We assume that 'energy lost' values in the table are dependent on the mass of the incoming particle and the thickness of the dead layer. In reality the "energy lost' values are also dependent on the energy of the incoming particle. The higher the energy of the incoming particle the less energy it will lose to the dead layer as it passes through. By not including this and assuming a particle loses the maximum amount of energy as it passes through; it means the very high energy particles are less affected by the correction but this is balanced as these particles are also less affected by the dead layer in the first place. For consistency the assumptions made for Table 1 are used throughout the process. Whilst this could be addressed by an iterative procedure, it is doubtful whether the effort would be justified given the inherent experimental uncertainties.

\section{Conclusions}

With the correction complete, the improvement to the accuracy of the data when considering the temporal changes in the dead layer thickness of the detector are clear. More importantly the method devised for correcting data is effective and can be easily adapted to other instruments, on other planetary missions containing silicon semi-conductor detectors. The final results present the data in a file for download (see Sect. 6), consisting of a before and after of the measured energy of the incoming particle combined with the original TOF readings and associated timestamp, for the entirety of the PHA high-rate data set.

The improvements to the data are advantageous as the correction of the energy not only improves our understanding of the energy densities to be expected in the Jovian magnetosphere, but also the ratios of the elements. This study offers an update to both the ratios of these elements, and also the energy distribution of the particles of each element. The secondary function of the correction method is useful for assigning each particle its most likely element, based on its new position.

For an inexpensive method, both in time and computation, the results of this correction are very useful for the Jovian magnetospheric community, and are available for use.

\section{Acknowledgments, Samples, and Data}

We thank Dr. Andreas Lagg for providing the PHA plotting routines and Dr. Norbert Krupp for useful discussions. The original as well as the corrected PHA event data are available from: http://sd-www.jhuapl.edu/Galileo_EPD/latest_calibrated_data/. Parts of the original data are also publicly available via the NASA archives (http://ppi.pds.nasa.gov/data/GO-JEPD-2-REDR-RTS-SCAN-AVG-V1.0/BROWSE/ (21/07/2016)). 
This work was supported by the Science Technology Research Council (grant number 1539671) and PEP Particle Environment Package for JUICE—Design for the Jupiter radiation environment (ST/N000161/1 2017-2018).

Publisher's Note Springer Nature remains neutral with regard to jurisdictional claims in published maps and institutional affiliations.

Open Access This article is licensed under a Creative Commons Attribution 4.0 International License, which permits use, sharing, adaptation, distribution and reproduction in any medium or format, as long as you give appropriate credit to the original author(s) and the source, provide a link to the Creative Commons licence, and indicate if changes were made. The images or other third party material in this article are included in the article's Creative Commons licence, unless indicated otherwise in a credit line to the material. If material is not included in the article's Creative Commons licence and your intended use is not permitted by statutory regulation or exceeds the permitted use, you will need to obtain permission directly from the copyright holder. To view a copy of this licence, visit http://creativecommons.org/licenses/by/4.0/.

\section{Appendix}

Table 2 Results from Chi Squared test of dead layer comparison accounting for the instrument calibration pre-mission launch. Bordered boxes correspond to the closest matching dead layer for each year

\begin{tabular}{lcclll}
\hline Chi $^{2}$ & $1996.272(35)$ & $1997.051(35)$ & $1999.18(28)$ & $2000.001(21)$ & $2002.925(28)$ \\
\hline $\begin{array}{l}\text { Calibration data } \\
\text { (originally } 0.075 \mu \mathrm{m})\end{array}$ & 2.184 & 6.209 & 8.384 & 9.970 & 14.211 \\
Dead layer $0.025 \mu \mathrm{m}$ & 2.943 & 3.174 & 5.432 & 6.455 & 9.959 \\
Dead layer $0.05 \mu \mathrm{m}$ & 7.790 & 3.873 & 3.820 & 4.447 & 7.3699 \\
Dead layer $0.075 \mu \mathrm{m}$ & 9.529 & 4.350 & 3.134 & 3.664 & 6.192 \\
Dead layer $0.1 \mu \mathrm{m}$ & 15.185 & 6.815 & 2.537 & 2.664 & 4.602 \\
Dead layer $0.125 \mu \mathrm{m}$ & 22.474 & 11.063 & 2.730 & 2.325 & 3.758 \\
Dead layer $0.15 \mu \mathrm{m}$ & 29.270 & 15.342 & 3.215 & 2.430 & 3.572 \\
Dead layer $0.175 \mu \mathrm{m}$ & 30.612 & 16.193 & 3.169 & 2.369 & 3.364 \\
Dead layer $0.2 \mu \mathrm{m}$ & 27.697 & 14.181 & 2.721 & 2.147 & 3.074 \\
Dead layer $0.225 \mu \mathrm{m}$ & 23.431 & 11.294 & 1.956 & 1.801 & 2.802 \\
Dead layer $0.25 \mu \mathrm{m}$ & 18.427 & 8.1809 & 1.613 & 1.809 & 2.965 \\
Dead layer $0.275 \mu \mathrm{m}$ & 32.323 & 17.065 & 2.886 & 2.158 & 2.714 \\
Dead layer $0.3 \mu \mathrm{m}$ & 21.665 & 10.080 & 1.548 & 1.591 & 2.537 \\
Dead layer $0.325 \mu \mathrm{m}$ & 34.707 & 18.5763 & 2.795 & 2.020 & 2.478 \\
Dead layer $0.35 \mu \mathrm{m}$ & 27.658 & 13.344 & 3.334 & 2.188 & 2.016 \\
Dead layer $0.375 \mu \mathrm{m}$ & 31.728 & 16.552 & 2.567 & 2.064 & 2.593 \\
Dead layer $0.4 \mu \mathrm{m}$ & 27.522 & 14.644 & 4.684 & 3.232 & 3.348 \\
Dead layer $0.5 \mu \mathrm{m}$ & 29.004 & 17.077 & 6.922 & 4.487 & 4.414 \\
\hline
\end{tabular}

\section{References}

F. Bagenal, The magnetosphere of Jupiter: Coupling the equator to the poles. J. Atmos. Sol.-Terr. Phys. 69(3), 387-402 (2007). https://doi.org/10.1016/j.jastp.2006.08.012

F. Bagenal, P.A. Delamere, Flow of mass and energy in the magnetospheres of Jupiter and Saturn. J. Geophys. Res. 116, A05209 (2011). https://doi.org/10.1029/2010JA016294 
F. Bagenal, T.E. Dowling, W.B. McKinnon, in Jupiter: The Planet, Satellites and Magnetosphere, ed. by F. Bagenal, T.E. Dowling, W.B. McKinnon. Cambridge Planetary Science, 1st edn. (Cambridge University Press, Cambridge, 2004)

E. Chané, J. Saur, S. Poedts, Modeling Jupiter's magnetosphere: Influence of the internal sources. J. Geophys. Res. Space Phys. 118(5), 2157-2172 (2013). https://doi.org/10.1002/jgra.50258

G. Clark, B.H. Mauk, C. Paranicas, P. Kollmann, H.T. Smith, Charge states of energetic oxygen and sulfur ions in Jupiter's magnetosphere. J. Geophys. Res. Space Phys. 121(3), 2264-2273 (2016). https://doi. org/10.1002/2015ja022257

C. Grupen, B. Shwartz, Particle Detectors (2011). https://doi.org/10.1017/CBO9780511534966

D.E. Huddleston, R.J. Strangeway, X. Blanco-Cano, C.T. Russell, M.G. Kivelson, K.K. Khurana, IO-Jupiter interaction: Waves generated by pickup ions. Adv. Space Res. 26(10), 1513-1518 (2000). Part of special issue: Planetary Ionospheres and Magnetospheres. https://doi.org/10.1016/s0273-1177(00)00091-0

I. Jun, H.B. Garrett, R. Swimm, R.W. Evans, G. Clough, Statistics of the variations of the high-energy electron population between 7 and 28 Jovian radii as measured by the Galileo spacecraft. Icarus 178(2), 386-394 (2005). https://doi.org/10.1016/j.icarus.2005.01.022

K.K. Khurana, Influence of solar wind on Jupiter's magnetosphere deduced from currents in the equatorial plane. J. Geophys. Res. Space Phys. 106(A11), 25999-26016 (2001). https://doi.org/10.1029/ 2000JA000352

B.H. Mauk, D.G. Mitchell, R.W. McEntire, C.P. Paranicas, E.C. Roelof, D.J. Williams et al., Energetic ion characteristics and neutral gas interactions in Jupiter's magnetosphere. J. Geophys. Res. Space Phys. 109(A9) (2004). https://doi.org/10.1029/2003ja010270

C. Paranicas, B.H. Mauk, K. Khurana, I. Jun, H. Garrett, N. Krupp, E. Roussos, Europa's near-surface radiation environment. Geophys. Res. Lett. 34(15) (2007). https://doi.org/10.1029/2007gl030834

D.J. Williams, Jupiter-At last! Johns Hopkins APL Tech. Dig. 17(4), 338-356 (1996)

D.J. Williams, Early energetic particle results from Jupiter. Johns Hopkins APL Tech. Dig. 18(2), 182-187 (1997)

D.J. Williams, R.W. McEntire, S. Jaskulek, B. Wilken, The Galileo energetic particles detector. Space Sci. Rev. 60(1-4), 385-412 (1992)

J.F. Ziegler, M.D. Ziegler, J.P. Biersack, SRIM-The stopping and range of ions in matter (2010). Nucl. Instrum. Methods Phys. Res., Sect. B, Beam Interact. Mater. Atoms 268(11-12), 1818-1823 (2010). https://doi.org/10.1016/j.nimb.2010.02.091 